\title{
Recuperación de espacios públicos. El caso del microcentro del centro histórico de San Salvador
}

Ana Cristina Vidal Vidales'

Julio César Martínez Rivera ${ }^{2}$

Recibido: 22/04/2013 - Aceptado: 13/09/2013

\section{Resumen}

La investigación realizada busca describir la utilización y apropiación de los espacios públicos del área denominada como microcentro, en la delimitación del centro histórico de San Salvador. Para ello, se utilizó la metodología cualitativa. Esta área presenta una complejidad de relaciones sociales conflictivas que han llevado a la degradación de los espacios públicos; a esto se suman diversos conflictos urbanos, así como el alcance de las acciones del gobierno municipal, a pesar de sus múltiples intentos por recuperar la zona.

\section{Palabras clave}

Espacios públicos, desarrollo de la comunidad urbanaSan Salvador, El Salvador, política urbana- San Salvador, El Salvador, rehabilitación urbana.

\section{Abstract}

This research seeks to describe the uses and forms of ownership of the public spaces located in the area known as micro-centre, in historical downtown of San Salvador. To do so, the qualitative method was used. This area in the historical downtown of San Salvador presents a complexity of conflictive social relationships that has led to the degradation of public spaces, to which diverse urban conflicts and the scope of the actions of the local government add up, despite its multiple efforts to recuperate the area.

\section{Keywords}

Public spaces, urban community development -San Salvador, urban policy-San Salvador.El Salvador, urban recovery, urban rescue.
El espacio público simboliza y contribuye al fomento de la ciudadanía e identidad nacional. Han tenido importancia plena a lo largo de la historia; así, las primeras ideas en torno a la concepción del espacio público surgen en Platón y Aristóteles, asociado al bienestar. Al llegar al siglo XVI, se concibe como algo compartido por todos, y luego se lo relaciona con el derecho (Neira, 2007). En tanto los espacios públicos no tienen un propietario privado, se asocian a la autoridad local más inmediata.
Sin embargo, dentro del urbanismo, se le ha considerado limitado a una dimensión meramente física. Algunos autores ven absolutamente necesario abordarlo desde su perspectiva histórica, bajo la comprensión de que el espacio público cumple una función socioeconómica crucial en las ciudades. Así, por ejemplo, se puede hablar de espacios privados de uso público, como los centros comerciales. El ágora griega, los mercados medievales y las plazas públicas latinoamericanas cumplieron esta función aglutinadora

1 Ana Cristina Vidal Vidales, arquitecta, con Maestría en Desarrollo Local, investigadora asociada de la Universidad Tecnológica de El Salvador, con experiencia en planificación estratégica participativa. acvidales@hotmail.com.

2 Julio César Martínez, licenciado en Trabajo Social, maestro en Administración de la Educación Superior, posgradro en Antropología Social, director de la Escuela de Antropología de la Universidad Tecnológica de El Salvador. julio.martinez@utec.edu.sv. 
de la población. Más que físico, el espacio público es un espacio de vinculación social, económica y política.

Los cambios producidos por la Revolución industrial a fines del siglo XIX, generan procesos de sobrepoblamiento de las ciudades, lo que necesariamente lleva a complejizar su estructura, sus espacios, su funcionamiento, las ocupaciones de la gente y su desarrollo económico. Tal y como lo indica Polése (citado en Granillo, 2009), es el desarrollo económico el que genera las ciudades y en una relación de doble dirección, las ciudades son necesarias para el desarrollo económico, aunque no son condición suficiente.

La introducción de nuevas tecnologías en la producción que se dio con la Revolución industrial condujo a un incremento del desempleo y a una mayor concentración de la riqueza; a una sobreexplotación del medio ambiente y desequilibrios territoriales. Todo esto impacta en la calidad de vida de la población y agudiza las desigualdades ya preexistentes. Existen, también, efectos esperados pero no deseados, como la migración del campo a la ciudad, que origina saturación en la demanda de los servicios y una enorme presión en las ciudades que buscan satisfacer las demandas de sus habitantes sin lograrlo plenamente. Efectos derivados son el hacinamiento y la violencia, la lucha por los recursos y la división social en el uso de los espacios públicos y privados de las ciudades.

Son las mismas condiciones de espacio, territorio y sociedad las que determinan la estructura social y física de una ciudad. Esa misma estructura tiene incidencia en los comportamientos, tanto colectivos como individuales; las relaciones sociales se vuelven superficiales (Elizalde \& Tijoux, 2008); el uso de los espacios se va transformando y los grandes espacios públicos de las élites cambian de lugar, del centro de la ciudad hacia los suburbios.

Los centros históricos, en general en Latinoamérica, se van degradando hasta lograr que una parte de la población, al menos, evite transitar por ese espacio. Los centros históricos son relevantes ciertamente porque tienen un valor histórico, patrimonial, cultural y, en algunos casos, hasta valor emocional. Sin embargo, más allá de esto se encuentra la identidad de una población. Por ello, la recuperación de los espacios debe servir para la generación de espacios para la sociedad, en donde la población lleve a cabo acciones donde se pueda socializar, recrearse, valorizarse y llegar a la plenitud espiritual (Rangel, 2002).

\section{El centro histórico de San Salvador: comercio, vivienda, movilidad, inseguridad y terremotos}

De acuerdo con el Foro permanente por el desarrollo integral del centro histórico de San Salvador (2010), más de la mitad del suelo del centro histórico está hoy ocupado por actividades de intercambio de bienes y servicios. Los espacios dedicados a la vivienda en el área son limitados y segregados para una población que cuenta con bajos recursos.

También se reconoce al centro histórico de la ciudad por su condición de inseguridad. Es por ello que los grandes comercios se retiran, minando sus capacidades de fortalecimiento y reproducción económica, a la vez, facilitando el apropiamiento de los espacios públicos por las ventas informales producidas por una desigual distribución de la riqueza; una disminución de las oportunidades de empleo en condiciones de satisfacción. También se detectan actividades ilícitas relacionadas o no con el comercio y grupos organizados delictivos. A pesar de esas características, el centro histórico sigue siendo un sitio donde convergen diariamente cientos de miles de salvadoreños. El transporte colectivo y el grueso de la población transita cotidianamente por la zona, lo que lo convierte en un espacio de privilegio para el comercio informal y en un sitio de alta contaminación ambiental, visual y acústica.

Por otro lado, la historia sísmica de la ciudad ha tenido un efecto importante en su infraestructura, ya que en la actualidad existen algunos edificios inhabitables; pero que llevan más de veinticinco años vacíos. La región donde se ubica la ciudad es de alta sismicidad. Así, ya en 1555, fray Antonio de Remesal anota un sismo mediano: "Se alborotó la tierra como si hubiera entrado en la villa todo el infierno junto" (Arias, 2007).

La ciudad, hoy, ha dejado de ser el centro de la política, de la economía y de las relaciones exteriores. El centro de Gobierno es disperso, las industrias y los centros comerciales están en el entorno de la ciudad y las embajadas se encuentran en la parte alta de San Salvador.

\section{Marco normativo del centro histórico de San Salvador}

El centro histórico de la ciudad cuenta con un amplio marco normativo que regula sus actividades. Este procede del gobierno local de la ciudad, y trata sobre los temas de participación ciudadana, convivencia ciudadana, mantenimiento y mejora de la infraestructura de los espacios públicos, uso del espacio público, conservación patrimonial, planificación, recaudación municipal e institucionalidad reguladora.

A esto se agrega la declaratoria legislativa a través del Decreto 680 del 18 de julio de 2008, publicado en el Diario Oficial Tomo 380, No. 155 (21 de agosto de 2008), en donde se reconoce la importancia cultural e histórica del área 
consolidada de la ciudad que actualmente se conoce con el nombre de centro histórico.

El inicio de los esfuerzos formales de planificación del área metropolitana de San Salvador (AMSS) se refieren al Plan regulador de 1954; al Plan vial metropolitano de 1956; al Nuevo plan regulador de 1961; al Plan de desarrollo de la Región Metropolitana de San Salvador (Metroplan) de 1968; al Metroplan de 1988; al Plan maestro de desarrollo urbano del área metropolitana de San Salvador (Plamadur-Amssa) de 1997; al Plan maestro del transporte salvadoreño (Plamatras) de 1995 ; al Plan nacional de ordenamiento y desarrollo territorial (PNODT) de 2001; al Plan San Salvador Ciudad del futuro del 2007, y al Plan de desarrollo territorial para la región metropolitana de San Salvador de 2011.

\section{Método}

Para llevar a cabo la investigación se utilizó la metodología cualitativa, a fin de describir la utilización de los espacios públicos en el microcentro del centro histórico de la ciudad de San Salvador.

Para ello, se tomaron datos por medio de tres técnicas de recolección: la realización de entrevistas a informantes clave, la realización de grupos focales, y visitas de campo.

\section{Participantes}

Se realizaron tres entrevistas a informantes claves:

1. Lic. Ismael Sermeño, como representante de la alcaldía municipal de San Salvador.

2. Arq. Raymundo Pineda, de la Fundación Salvadoreña de Desarrollo y Vivienda Mínima (Fundasal).

3. Sr. Pedro Julio Hernández, representante del sector del comercio informal en el centro histórico de San Salvador a través de la Coordinadora Nacional de Vendedores (CNV).

Adicionalmente, se realizaron dos grupos focales:

1. Con personas que visitan regularmente el centro histórico de San Salvador y tienen una opinión formada acerca de sus propias percepciones de la situación.

2. Con vendedores del sector informal, con el fin de conocer su posición más en profundidad.

\section{Instrumentos}

Para la recolección de datos cualitativos se confeccionaron guías de preguntas. Cada una de las entrevistas y grupos focales contó con su propio instrumento.
Para la realización de un mapa de usos del suelo del centro histórico se retomó el instrumento diseñado por Fundasal y el Foro Permanente por el desarrollo integral del centro histórico de San Salvador, en su publicación Usos del suelo en el centro histórico de San Salvador. Se utilizó esta ficha de usos del suelo con el fin de actualizar la información, debido a que el uso que se hace de los espacios en el área de estudio ha sido dinámico, en parte debido a las acciones que el gobierno local ha estado implantando en sus esfuerzos por recuperar el espacio público.

\section{Procedimiento}

El material y los datos obtenidos por medio de la recolección cualitativa fue grabado, con el conocimiento de los participantes, y los archivos de audio posteriormente transcritos para luego hacer la sistematización de la información obtenida por medio de matrices que facilitaran el análisis.

La información recolectada por medio de las fichas de usos del suelo fue organizada y presentada en un plano del área en estudio.

Posteriormente, toda la información fue analizada y contrastada para presentar los distintos puntos de vista de los participantes.

\section{Resultados}

\section{Los usos del suelo en el microcentro del centro histórico de San Salvador}

El uso del suelo en el centro histórico de San Salvador es fundamentalmente comercial, coexistiendo lo formal y con lo informal en toda el área. Los vendedores de la calle conocen dónde están los compradores y por eso usan el centro de la ciudad, sitio de tránsito de grandes sectores populares, como un mercado al aire libre.

Económicamente, la venta en la calle es el reflejo de una situación de gravedad; estéticamente, no resulta agradable para vivir en su entorno; axiológicamente, degrada el valor del espacio; políticamente, demuestra ingobernabilidad. Ello ha llevado a perder la función de habitabilidad del centro de la ciudad, y lo usan como espacio habitacional solamente quienes tienen recursos más limitados.

Hasta hoy, las plazas públicas se han logrado mantener relativamente despejadas de comercio informal. Sin embargo, es muy probable encontrar siempre comercio informal en este entorno. 
Para la Fundasal, la recuperación de la función de vivienda del centro de la ciudad es un elemento central, trabajando en los aspectos sociales y considerando su cultura a efectos de promover ambientes congruentes con la población, que participa organizadamente en la gestión de sus propios proyectos habitacionales; eso les da una nueva autoestima.
La organización de cooperativas de vivienda compromete a sus socios en los objetivos y fines de contar con habitaciones limpias y adecuadas, y existe una preocupación para que el paisaje social inmediato se mantenga en forma de sana convivencia, a partir de las autorregulaciones promovidas por la población a través de sus representantes organizados.

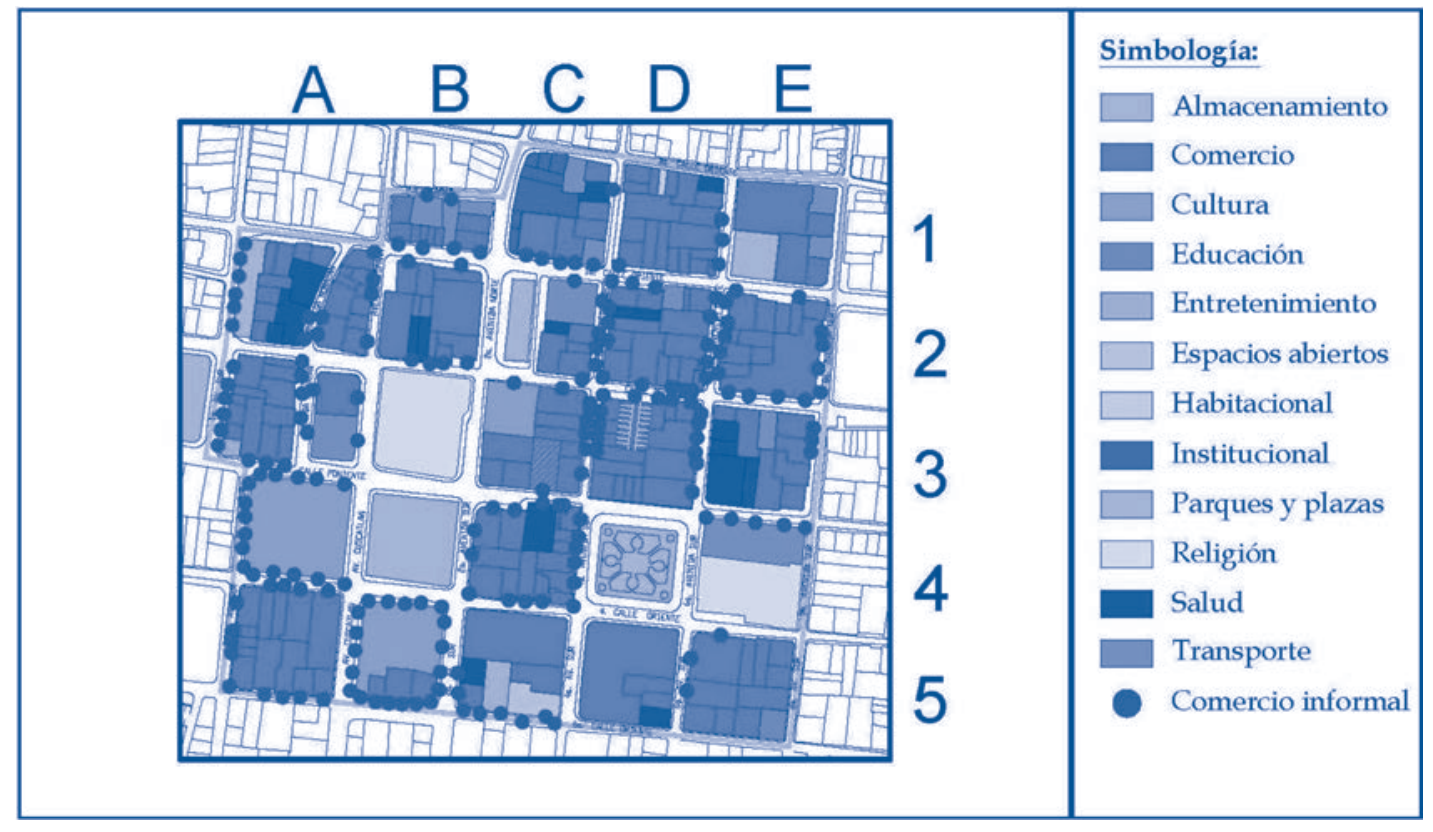

Imagen 1. Usos de suelo en el microcentro del centro histórico de San Salvador

Fuente: Elaboración propia con base en ficha de recolección de datos realizada por alumnos ayudantes de investigación supervisados por el Lic. Julio Martínez.

Uno de los proyectos de Fundasal, en el barrio San Esteban, se trabajó con diseños participativos, en donde confluyeron intereses distintos entre el grupo, que se resolvieron por la vía de la negociación entre ellos, dando y recibiendo. El centro de la ciudad se ha vuelto, para quienes lo habitan, su propio espacio identitario, aun cuando, para quienes viven fuera de él, sea solamente un sitio de paso.

Las concepciones acerca de cómo se usa el espacio público no escapan a contradicciones entre sus usuarios. Así, los comerciantes informales consideran que, a pesar de las ofertas electorales que llegan de distintas orientaciones políticas, estas no se cumplen; y que el espacio público es "la única fábrica de empleos que tienen las familias pobres de este país". Se consideran a sí mismos perseguidos, reprimidos y criminalizados por utilizar el espacio público en el centro de la ciudad. Sin embargo, hacen notar que otros espacios públicos son utilizados por otros sectores y no hay represión hacia estos. Entre esos se mencionan las playas.
Desde la perspectiva de los representantes del comercio informal, es necesario asumir la causa estructural de la situación y, a partir de ello, juntar a los diversos sectores para encontrar las soluciones. "Polvos azules", un centro comercial de informales desarrollado como experiencia exitosa en Lima, Perú, es un referente sobre cómo deberían hacerse las cosas. Desde su punto de vista, el comercio formal se vincula con ellos para la distribución de sus productos, lucrándose, mientras ellos continúan sumergidos en la pobreza. Eso es una enorme lección de economía que las personas que se dedican al comercio informal han aprendido en la práctica.

Hay dos grandes consideraciones sobre la situación, desde su comprensión:

1. Los grandes centros comerciales reducen sus oportunidades de negocio. Esto está también sujeto a análisis, ya que muchas personas consideran que los centros comerciales dan seguridad, estética, limpieza y 
orden. Aparte de su publicidad, estos parecen ser los factores que atraen en un centro comercial.

2. El centro histórico no es más importante que su propia necesidad de sobrevivencia, aunque es aceptado que ha habido casos en que algunos comerciantes informales han sido sucios, descuidados y abusivos. También se reconoce su incapacidad para poder visibilizar su propio drama. No han sido capaces de dar respuestas contundentes y sin violencia hacia las otras propuestas y acciones de otros actores que, a su juicio, son cargadas de injusticia e inequidad.

\section{Discusión}

La venta informal en la calle es una cuestión que se puede vincular con los últimos años del siglo XIX. Antes de eso, las plazas eran los sitios de comercio. Se entendía que los "días de plaza" se relacionaban con la libertad y oportunidad de vender en esos espacios que, en otros días, eran limitados al ocio y esparcimiento. Las necesidades de la población de comprar más, e incluso diariamente, promueven la necesidad de contar con esa infraestructura que hoy llamamos mercado, y que, en el caso de San Salvador, ha llegado a contar con mercados especializados como el Emporium (flores), el Número 5 (carnes), de herramientas (Belloso) y otros, algunos ya desaparecidos.

A pesar de eso, la industrialización del país, la creación de importantes polos de desarrollo industrial representados en el bulevar del Ejército, a fines de los años 50 e inicios de los 60 (Rodríguez, 2002), sirvió como gran atractivo para la migración del campo a la ciudad. Estos migrantes no siempre encontraron un empleo y debieron dedicarse a comerciantes en la calle para subsistir.

En los años 70, se desarrollan actividades de protestas en la calle y los espacios públicos se saturaron; y el problema desborda las soluciones que se puedan encontrar en el municipio que amplía la infraestructura de mercados y crea zonas peatonales, libres del tránsito de vehículos. Se continúa planificando la creación de nuevos centros de comercio en el centro de la ciudad para abastecer las necesidades diarias. En los años 80 se amplían las zonas peatonales y nuevamente se incrementa la infraestructura de mercados. Sin embargo, eso no soluciona los problemas del uso del espacio público en la ciudad, que con la llegada del conflicto utiliza los recursos de ordenamiento hacia la atención de la subversión.
Los acuerdos entre comerciantes informales, el municipio y el sector formal del comercio no han sido alcanzados de manera efectiva ni conveniente para identificar y apoyar una respuesta satisfactoria a la problemática.

Desde la perspectiva de los informales, las soluciones, en el caso de El Salvador, no son suficientemente atractivas. El uso de antiguos cines convertidos en centros de comercio informal no es adecuado, ya que el cine está diseñado para evitar la visibilidad hacia el interior, y las adecuaciones realizadas no son suficientes. Otras estrategias son menos felices, como los desalojos por la vía de la fuerza, en los que incluso han resultado fallecidos en los enfrentamientos. La estrategia del desalojo, por otra parte, genera alta tensión social.

Desde la posición de los informales, las soluciones buscadas por el poder local solo se enmarcan en una perspectiva cosmética del problema, en lugar de propiciar soluciones estructurales, dicho por ellos: "Quienes dan vida al centro histórico es la gente, no los edificios".

Desde la visión de ellos, una de las variables importantes en la resolución es la vinculación y coordinación entre el gobierno central y el local, haciendo notar que hasta hoy las ideas de los dos niveles de gobierno son incompatibles. Los informales también han planteado propuestas, como la creación de otros centros comerciales y estacionamientos en predios como el Hula Hula, el ex Universidad Nacional y el ex Biblioteca Nacional.

Otras soluciones atraviesan por la reorientación de las actividades, la generación de nuevas competencias para que los informales puedan dedicarse a otras actividades y así lograr la recuperación del espacio público.

La presión de la municipalidad es, a veces, ejercida desde los medios de comunicación. En octubre de 2012, a sabiendas de que nadie lo poseía, se publicó que los vendedores del microcentro tenían tres días para presentar su permiso de comercio o abandonar sus actividades comerciales en el sector, bajo el amparo de la ordenanza reguladora del comercio informal.

Esto fue el origen de nuevos conflictos y se realizó una de las limpiezas más radicales del área: enfrentamientos armados, conatos de incendio, restricción de acceso a la zona, limpieza y otras acciones importantes. Una semana más tarde, los comerciantes informales estaban de nuevo apostados en sus antiguos sitios, excepto aquellos a quienes se les había eliminado la infraestructura. 


\section{Causas y efectos del estado actual de los espacios públicos del microcentro del centro histórico de San Salvador}

Evidentemente, las causas de la problemática de la degradación de los espacios públicos en el centro histórico de San Salvador son de carácter estructural: pobreza, falta de empleo y baja inversión para la producción. Los que tienen menos oportunidades sufren la exclusión social, y se provocan, además, oportunidades delictivas.

Una buena parte de los espacios públicos no recibe una atención integral del Estado. Ello es particularmente cierto para el microcentro del centro histórico en San Salvador. Por otro lado, no parece existir un presupuesto asignado para la recuperación del patrimonio, aunque existe normativa suficiente. A esto se le agrega el desinterés de la población por su propio patrimonio, como resultado de procesos educativos deficitarios. Experiencias de otros países hacen notar cómo el Estado invierte en la recuperación del patrimonio histórico y cultural de sus ciudades.

Existe, además, la percepción de que la construcción de nuevos espacios afecta el medio ambiente en la medida en que se vuelven espacios artificiales; y luego está el hecho de una muy limitada intervención de las autoridades locales en la conservación del patrimonio.

La población que asiste al centro histórico como un lugar de confluencia, de tránsito y en menor medida por las actividades culturales que se desarrollan y a ejercer sus compras, anotan, además, que el mayor problema es la inseguridad que afecta más a mujeres que a hombres. El centro de la ciudad es percibido como un sitio muy peligroso, favorecido por un alto tránsito de rutas de buses, estaciones y paradas, con hacinamiento y propicio para los actos delictivos.

Los usuarios transeúntes del centro histórico de San Salvador perciben que la responsabilidad del espacio público, en lo referente a mantenimiento, orden y seguridad, es del Estado y del gobierno local. Aunque la ciudadanía tiene también parte de responsabilidad en lo referido a orden y limpieza.

\section{Conclusiones y recomendaciones}

1. Para el éxito de los proyectos en torno al centro histórico de San Salvador es imprescindible la organización y las redes, con papeles definidos.

2. La recuperación del centro histórico implica la participación de la población. Este es un asunto clave.
Esta participación debe ser representativa, de calidad, y debe aportar insumos e ideas productivas.

3. Es necesaria la revisión y articulación de las actuales normativas para subsanar omisiones, duplicidades y ambigüedades, y profundizar el tema de la participación de la población.

4. La generación del arraigo identitario permite que las personas refuercen la relación que tienen con los espacios públicos. El reencuentro con la memoria histórica para la realización de actividades colectivas fortalece los vínculos de las organizaciones con su espacio geográfico, y, por lo tanto, les permite dedicarse al mantenimiento de las tradiciones. Se debe, pues, desarrollar un mayor número de actividades culturales en la ciudad.

5. La visión a largo plazo es necesaria porque permite la consecución progresiva de los objetivos planteados.

\section{Referencias}

Aledo, A. (2000). El significado cultural de la plaza hispanoamericana. El ejemplo de la plaza mayor de Mérida. Revista Tiempos de América (5-6), 37-47.

Arias, J. (2007). San Salvador, ciudad de 450 años. (G. Ramirios, Ed.) San Salvador: centro de Promoción Cultural y Recreativa Municipal de San Salvador.

Elizalde, A. \& Tijoux, M.E. (2008). Ciudad: Espacios y flujos. Polis, Revista de la Universidad Bolivariana (020).

Foro permanente por el desarrollo integral del centro histórico de San Salvador. (2010). Usos del suelo en el centro histórico de San Salvador. San Salvador: Impresos Quijano.

García Canclini, N. (1990). Culturas híbridas. Estrategias para entrar y salir de la modernidad. México, D.F.: Grijalbo.

Munizaga, G. (2000). Macroarquitectura. Tipologías y estrategias de desarrollo urbano (Segunda ed.). México, D.F.: Alfaomega Grupo Editor.

Neira, H. (2007). La naturaleza del espacio público. Una visión desde la filosofía. En O. Segovia (Ed.), Espacios públicos y construcción social. Hacia un ejercicio de ciudadanía pp. 29-40. Santiago de Chile: Ediciones SUR.

Rangel, M. (Agosto de 2002). La recuperación del espacio público para la sociabilidad ciudadana. Recuperado el 3 de abril de 2012, de sitio web de Universidad de Los Andes, Venezuela: http://www.saber.ula.ve/ bitstream/123456789/13458/1/recuperacion_spacio. pdf

Rodríguez, A. (2002). San Salvador, historia urbana 19001940. San Salvador: Dirección de Publicaciones e Impresos. 